\title{
Avaliação da acurácia planimétrica das imagens do Google Earth para produção de base cartográfica
}

\author{
for cartographic base production \\ Raiane Rintielle Vaz Menezes ${ }^{1}$ \\ Matheus Henrique Maia Lisboa ${ }^{2}$ \\ Afonso de Paula dos Santos ${ }^{3}$ \\ Jairo dos Santos Dias ${ }^{4}$
}

Evaluation of the planimetric accuracy of Google Earth

Recebido em dezembro de 2018.

Aprovado em abril de 2019.

\begin{abstract}
RESUMO
Atualmente, vivencia-se uma expansão de novas tecnologias de mapeamentos e de compartilhamento de informações geoespaciais. Uma dessas tecnologias é o Google Earth, na qual usuários têm acesso às informações cartográficas. Porém seu uso geralmente é feito sem o conhecimento sobre a qualidade posicional das imagens utilizadas. Neste sentido, o presente estudo tem como objetivo avaliar a qualidade das imagens do Google Earth para produção de base cartográfica digital. A avaliação foi realizada com base na acurácia posicional, a partir de testes estatísticos, seguindo a metodologia proposta por Santos et al. (2016), a qual sugere a análise do padrão de distribuição espacial, a detecção de outliers, a análise de normalidade e a análise de tendência dos dados de teste, respeitando os critérios instituídos pelo Decreto $n^{\circ}$ 89.817/1984, e aliada às especificações ET-ADGV (DSG, 2011) e ET-CQDG (DSG,2016). Apesar de as imagens advindas do Google Earth apresentarem deslocamentos quanto às posições referências, após a correção dessas tendências, os resultados obtidos apresentaram uma classificação compatível com a Classe A (PECPCD), na escala 1/25.000, e Classe B (PEC-PCD), para a escala 1/10.000.
\end{abstract}

PALAVRAS-CHAVE: Google Earth. Base Cartográfica. Controle de Qualidade Cartográfica.

\footnotetext{
${ }^{1}$ Universidade Federal de Viçosa, Departamento de Engenharia Civil. Programa de Pós-graduação em Engenharia Civil, Brasil. E-mail: raiane.menezes@ufv.br

2Universidade Federal de Viçosa, Departamento de Engenharia Civil. Programa de Pós-graduação em Engenharia Civil, Brasil. E-mail: lisboamhm@gmail.com

${ }^{3}$ Universidade Federal de Viçosa, Departamento de Engenharia Civil. Programa de Pós-graduação em Engenharia Civil, Brasil. E-mail: afonso.santos@ufv.br

${ }^{4}$ Universidade Federal de Viçosa, Departamento de Engenharia Civil. Programa de Pós-graduação em Engenharia Civil, Brasil. E-mail: jairo.dias@ufv.br
} 


\begin{abstract}
Currently, we are experiencing an expansion of new mapping and geospatial information sharing technologies. One of these technologies is Google Earth, through which users have access to cartographic information. However, its use is usually done without the knowledge about the positional quality of the images. In this sense, the present study aims to evaluate the quality of the Google Earth images mosaic for production of digital cartographic base. The evaluation was performed based on the positional accuracy, from statistical tests were used, by following the methodology proposed by Santos et al. (2016), which suggests the analysis of the spatial distribution pattern, outliers detection, normality analysis and trend analysis of the test data, respecting the criteria established by Decree $\mathrm{N}^{\circ}$ 89.817/1984, and allied to ET-ADGV (DSG, 2011) and ET-CQDG (DSG, 2016). Although the images from Google Earth show displacements regardind the reference positions, after the correction of the trends, the results presented a classification compatible with Class A (PEC -PCD) on 1 / 25.000 scale and Class B (PEC-PCD) to 1 / 10.000 scale.
\end{abstract}

KEYWORDS: Google Earth. Cartographic Base. Cartographic Quality Control.

$$
* * *
$$

\title{
Introdução
}

O desenvolvimento e a importância da cartografia originam-se da necessidade do ser humano em se localizar e se orientar no espaço. Posteriormente, o conhecimento da cartografia torna-se um importante instrumento de conquista de territórios, de forma que o avanço desta ciência se torna fundamental para o desenvolvimento dos Estados (SANTOS, 2013).

$\mathrm{Na}$ atualidade, vivencia-se uma revolução geoespacial, em que a tecnologia utilizada para o mapeamento se desenvolve espantosamente (SANTOS et al., 2016). As informações cartográficas passaram a ser parte do cotidiano da sociedade, tais como a utilização de mapas e imagens orbitais, disponíveis em diversas plataformas, como Google Earth, Google Maps, Bing Maps, OpenStreetMap e outros; de receptores GNSS (Global Navigation Satellite System), disponíveis em celulares; e de sistemas de roteamento e navegação terrestres para automóveis. Atualmente a informação espacial pode ser produzida e apresentada por usuários comuns que tenham acesso a um computador ou smartphone e à internet (BRAVO e SLUTER, 2015). 
Nesse contexto, tem-se o Google Earth, uma das geotecnologias mais difundidas na atualidade, caracterizada como uma plataforma gratuita que permite ao usuário navegar por todo o globo terrestre. É um software desenvolvido e distribuído pela empresa Google, o qual apresenta um modelo tridimensional da superfície terrestre. Constitui-se de um mosaico de diversas imagens orbitais, que são frequentemente atualizadas, porém não são disponibilizadas em tempo real (SCHMITT et al., 2013). A partir das imagens do Google Earth, é possível identificar lugares, construções, cidades, paisagens, entre outros elementos (PAIVA, 2010).

Com toda esta disponibilidade e facilidade de acesso à geoinformação, muitos usuários utilizam indiscriminadamente essas fontes de dados, sem saber ao certo se são confiáveis, desconhecendo, por exemplo, qual o nível de acurácia posicional e acurácia temática e qual a temporalidade dos produtos disponibilizados. Bravo e Sluter (2015) comenta que devido a essa problemática, instituições, como a Organização Internacional para Padronização (ISO), desenvolvem e estudam normas específicas para a adequação das avaliações da qualidade dos dados espaciais. Uma maneira de avaliar estes produtos é aplicar o controle de qualidade cartográfica. Dentre os elementos presentes no controle de qualidade, a acurácia posicional é um dos mais estudados e avaliados (ARIZA, 2002; NOGUEIRA, 2003; SANTOS, 2010).

Conforme Galo e Camargo (1994), a avaliação da acurácia posicional de um produto ou dado cartográfico se baseia na análise das discrepâncias entre as coordenadas dos pontos retirados no produto e seus homólogos obtidos a partir de observações realizadas em campo ou por meio de um produto mais acurado, neste caso chamados de referência. Para Merchant (1982) e Tommaselli et al. (1988), os pontos de referência devem apresentar uma incerteza inferior a um terço do erro padrão esperado para o produto.

Goodchild (2010) ressalta a importância do controle de qualidade ao investigar dados espaciais, de modo a garantir e especificar a qualidade e a 
acurácia deles, explicitando discrepâncias, omissões e incertezas, bem como definindo sua finalidade.

A avalição da acurácia posicional de produtos cartográficos, no Brasil, segue as diretrizes do Decreto $n^{\circ} 89.817$ (BRASIL, 1984) que classifica um produto em três classes, A, B e C, baseado nas tolerâncias PEC e EP para uma determinada escala.

No Brasil, o estudo da acurácia posicional de imagens provenientes da plataforma do Google Earth vem sendo explorado em diversas regiões, de forma crescente, desde o lançamento da ferramenta em 2001, destacando-se que os trabalhos mais citados foram produzidos nos últimos 10 anos.

Silva e Nazareno (2009) avaliaram a acurácia posicional de imagens do Google Earth, no município de Goiânia/GO, por meio do teste do QuiQuadrado, do que resultou a classificação da acurácia posicional como classe A, para a escala 1/5.000. Foi identificada uma tendência no produto, e o deslocamento foi corrigido a partir de uma subtração da média das discrepâncias calculadas para cada uma das direções, Este e Norte, pelas coordenadas obtidas nas imagens. Oliveira et al. (2009) avaliaram um mosaico elaborado pela captura de cenas disponibilizadas pelo Google Earth, para todo o município de São Leopoldo/RS. Os autores utilizaram o teste do Qui-Quadrado para a avalição da precisão e o teste t de Student para a exatidão e concluíram que, para a região de estudo, as imagens não apresentaram tendências e eram compatíveis com a Classe B para a escala $1 / 15.000$.

Já Soares et al. (2010) avaliaram imagens do Google Earth para a zona urbana de Pato Branco/PR, através do teste do Qui-Quadrado. O produto teste apresentou tendência e foi compensado, resultando em um padrão classe A para a escala 1/30.000. Destaca-se que o processo de validação dos trabalhos citados foi baseado na análise das discrepâncias entre as coordenadas obtidas através de receptores GNSS e aquelas obtidas nas imagens do Google Earth. A análise de tendência foi baseada na distribuição t de Student, e a análise de precisão, baseada na distribuição Qui-quadrado. Lopes (2009) avaliou a 
qualidade posicional e geométrica das imagens de alta resolução disponibilizadas pelo Google Earth, para o município de Barbacena/MG. Utilizou-se como referência uma base de dados municipal na escala 1/2.000, onde comparou 12 pontos de checagem. Os resultados indicaram a fidelidade geométrica de imagens do Google Earth, em comparação ao mapeamento do município de Barbacena, porém apresentaram tendência na avaliação. Para classificação da imagem, o autor utilizou como base o erro de graficismo (0,2 $\mathrm{mm}$ ), por meio do qual encontraram uma escala de aproximadamente $1 / 50.000$.

A partir dos resultados destes trabalhos, observa-se que as acurácias posicionais das imagens desta plataforma variam de região para região e que, apesar de todos os autores seguirem a mesma metodologia (teste do QuiQuadrado), todos apresentaram formas distintas para sua aplicação, não havendo uma padronização. Ressalta-se que recentemente foi lançada a Especificação Técnica para Controle de Dados Geoespaciais (ET-CQDG2016), que apresenta uma metodologia mais detalhada para classificação dos produtos cartográficos. Além disso, o Manual Técnico em Geociências, número 13 (Avaliação da Qualidade de Dados Geoespaciais), publicado pelo IBGE (2017) acrescenta mais informações sobre o processo de análise de qualidade em produtos cartográficos.

Santos et al. (2016) apresentam uma metodologia para a classificação de produtos cartográficos, incorporando técnicas da estatística espacial, como a análise do padrão de distribuição espacial e a análise de tendências na amostra de discrepâncias posicionais, as quais, aliadas à metodologia da ETCQDG (2016), proporcionam uma avaliação mais completa e segura do produto.

Diante do exposto, o objetivo deste trabalho é avaliar a qualidade das imagens do Google Earth para a utilização na produção de base cartográfica, a partir da acurácia posicional planimétrica, de acordo com a metodologia descrita pelo Decreto n 89.817 (BRASIL, 1994), pela ET-CQDG (2016) e por Santos et al. (2016). 
Vergara, Cintra e D'Alge (2001) destacam que apesar de todos os avanços tecnológicos, muitas regiões do Brasil sofrem com a falta de mapeamento cartográfico sistemático, a desatualização das bases existentes e uma carência de bases cartográficas em grandes escalas. Desta forma, considera-se também neste estudo a verificação da possibilidade de utilização das imagens do Google Earth para uma atualização do mapeamento sistemático brasileiro, em escalas pequenas a médias (1/1.000.000, 1/500.000, $1 / 250.000,1 / 100.000,1 / 50.000$ e $1 / 25.000)$.

\section{Padrão Brasileiro de acurácia posicional}

A qualidade de um produto cartográfico deve ser garantida por normas e leis que especifiquem os parâmetros mínimos de confiabilidade. A norma brasileira para regulamentar produtos de natureza cartográfica foi criada pela Comissão Nacional de Cartografia (CONCAR), em 1984, na forma do Decreto $\mathrm{n}^{\circ}$ 89.817, de 20 de junho de 1984 (BRASIL, 1984), que estabelece as Instruções Reguladoras das Normas Técnicas da Cartografia Nacional e define as tolerâncias do Padrão de Exatidão Cartográfica (PEC) e do ErroPadrão (EP). Em seu artigo $9^{\circ}$, define as tolerâncias para três classes possíveis de enquadramento, na avaliação da acurácia posicional em dados cartográficos (Tabela 1). Ressalta-se que as tolerâncias, para a planimetria, dependem da escala.

Tabela 1- Valores do PEC e do EP segundo Decreto n 89.817 (Brasil, 1994)

\begin{tabular}{c|c|c}
\hline \multirow{2}{*}{ Classe } & \multicolumn{2}{|c}{ Planimetria } \\
\cline { 2 - 3 } & PEC (mm) & EP (mm) \\
\hline $\mathrm{A}$ & 0,5 & 0,3 \\
\hline $\mathrm{B}$ & 0,8 & 0,5 \\
\hline $\mathrm{C}$ & 1,0 & 0,6 \\
\hline
\end{tabular}

Em 2011, foram publicadas as Especificações Técnicas de Aquisição de Dados Geoespaciais Vetoriais (ET-ADGV), pela Diretoria do Serviço Geográfico do Exército Brasileiro (DSG, 2011). Tal especificação explica como 
deve ser a aplicação do Decreto $\mathrm{n}^{\circ}$ 89.817, bem como cria uma classe para produtos cartográficos digitais (PEC-PCD), com tolerância mais restritiva. Cintra e Nero (2005) afirmam que independentemente de o produto ser digital ou analógico, sua validação pelo Padrão de Exatidão Cartográfica (PEC) é a mesma.

A Tabela 2 apresenta as tolerâncias utilizadas na avaliação da acurácia posicional utilizando o padrão descrito na ET-ADGV (DSG, 2011), utilizando como base o Decreto nº 89.817 (BRASIL, 1994).

Tabela 2- Classificação da acurácia planimétrica conforme ET-ADGV (DSG, 2011)

\begin{tabular}{c|c|c}
\hline \multirow{2}{*}{$\begin{array}{c}\text { Classe } \\
\text { PCD }\end{array}$} & PEC (mm) & EP (mm) \\
\cline { 2 - 3 } A & 0,28 & 0,17 \\
\hline $\mathrm{B}$ & 0,5 & 0,3 \\
\hline $\mathrm{C}$ & 0,8 & 0,5 \\
\hline $\mathrm{D}$ & 1,0 & 0,6 \\
\hline \multicolumn{2}{c}{ Fonte: Elaborada pelos autores. }
\end{tabular}

Já em 2016, a Norma da Especificação Técnica para Controle de Qualidade de Dados Geoespaciais (ET-CQDG), baseada na ISO 19157/2013, foi publicada pela Diretoria de Serviço Geográfico do Exército Brasileiro (DSG, 2016). Esta especificação padroniza a metodologia utilizada na avaliação da qualidade dos produtos de conjuntos de dados geoespaciais, permitindo comparar conjuntos de dados avaliados por uma estrutura comum. A ET-CQDG (DSG, 2016) também define uma metodologia a ser utilizada para avaliar a acurácia posicional em produtos cartográficos, baseada nos preceitos do Decreto n 89.817 (BRASIL, 1994) e na ET-ADGV (DSG, 2011). Assim, a ET-CQDG estabelece que os critérios a e b, a seguir, devem ser atendidos simultaneamente:

a) $90 \%$ dos pontos coletados no produto, em relação aos seus pontos homólogos de maior precisão, não deverão apresentar discrepâncias superiores ou iguais ao PEC;

b) O valor Root Mean Square (RMS) da amostra de discrepâncias deverá ser igual ou inferior ao valor do erro padrão (EP). 


\section{Materiais e métodos}

2.1 Área de estudo

A área de estudo selecionada foi o município de Paula Cândido, localizado na Zona da Mata do estado de Minas Gerais. Possui uma área de $268,39 \mathrm{~km}^{2}$, delimitada pelas latitudes $20^{\circ} 45^{\prime} 18^{\prime \prime}$ 'S e $20^{\circ} 59^{\prime} 08^{\prime \prime}$, e longitudes $43^{\circ} 08^{\prime} 55^{\prime} \mathrm{W}$ e $42^{\circ} 52^{\prime} 56^{\prime} \mathrm{W}$, conforme apresentado na Figura 1. A região de estudo foi escolhida devido ao fato de ser um município de pequeno porte e de se ter a possibilidade de levantamento in loco dos pontos de referência.

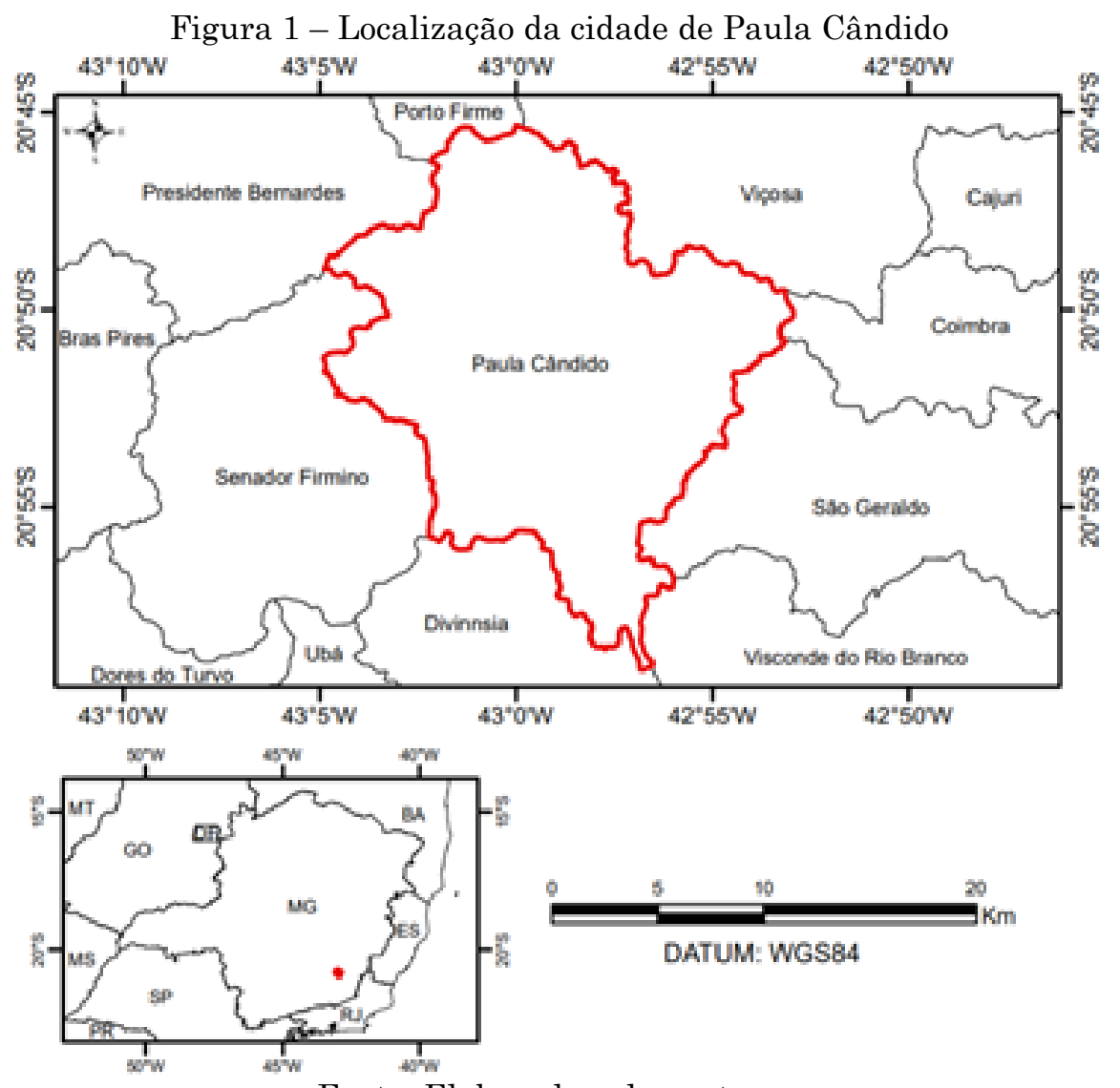

Fonte: Elaborada pelos autores. 


\subsection{Materiais utilizados}

Para o desenvolvimento deste estudo, foi utilizado um mosaico de imagens, datadas de agosto de 2014 a maio de 2017, obtidas pelo Google Earth, do município de Paula Cândido/MG.

Foi utilizado o software ArcGIS v.10.4 para o planejamento da distribuição das amostras e a análise da distribuição espacial. Utilizou-se o software GeoPEC v. 3.4, disponibilizado gratuitamente, para a avaliação da Acurácia Posicional Planimétrica e para a classificação de acordo com o Decreto n 89.817 (BRASIL, 1994) e com a ET-CQDG (DSG, 2016).

Os pontos de verificação foram coletados a partir do receptor GNSS da marca TopoMap, modelo T10 de dupla frequência. O posicionamento foi realizado pelo método relativo estático com rastreamento de $20 \mathrm{~min}$., considerando como base a estação VICO, situada na cidade de Viçosa/MG, pertencente à Rede Brasileira de Monitoramento Contínuo dos Sistemas GNSS (RBMC-IBGE). O processamento dos dados foi efetivado no software Topcon Tools 7.5.1. A maior linha de base utilizada foi da ordem de $25 \mathrm{Km}$. A precisão posicional planimétrica obtida no levantamento apresentou valores de $1 \mathrm{~cm}$ a $33 \mathrm{~cm}$. Tanto as coordenadas do mosaico do Google Earth quanto as dos pontos de verificação se encontram referenciadas ao Datum WGS84, com sistema de projeção UTM do fuso 23 Sul.

\subsection{Metodologia}

Neste estudo foram utilizadas as metodologias descritas pela ETCQDG (DSG, 2016), por Santos et al. (2016) e pelo Manual do IBGE (2017). A Figura 2 apresenta a metodologia executada. A primeira etapa para avaliar a acurácia posicional é ter uma amostra de pontos de checagem que sejam bem definidos e distribuídos espacialmente sobre o dado em avaliação. A definição do tamanho amostral foi realizada conforme as normativas ET-CQDG (DSG, 2016) e IBGE (2017), todas baseadas nas especificações da ISO 2859-1 e ISO 
2859-2. Estas trazem o conceito de nível de inspeção, que discrimina a quantidade relativa de inspeção em uma população, dividido em três níveis gerais: o nível III, mais rigoroso, o nível II e o nível I, menos rigoroso. Essas normas também trazem o conceito de Acceptance Quality Limit (LQA), que estabelece o pior resultado possível dentro dos limites aceitáveis de qualidade, para o processo de Lote a Lote (quando se tem 10 ou mais lotes em avaliação); bem como o conceito de Limiting Quality (QL) que representa um nível de qualidade para um lote isolado (quando se tem menos que 10 lotes em avaliação) limitado a uma baixa probabilidade de aceitação (DSG, 2016).

Figura 2 - Fluxograma da metodologia aplicada
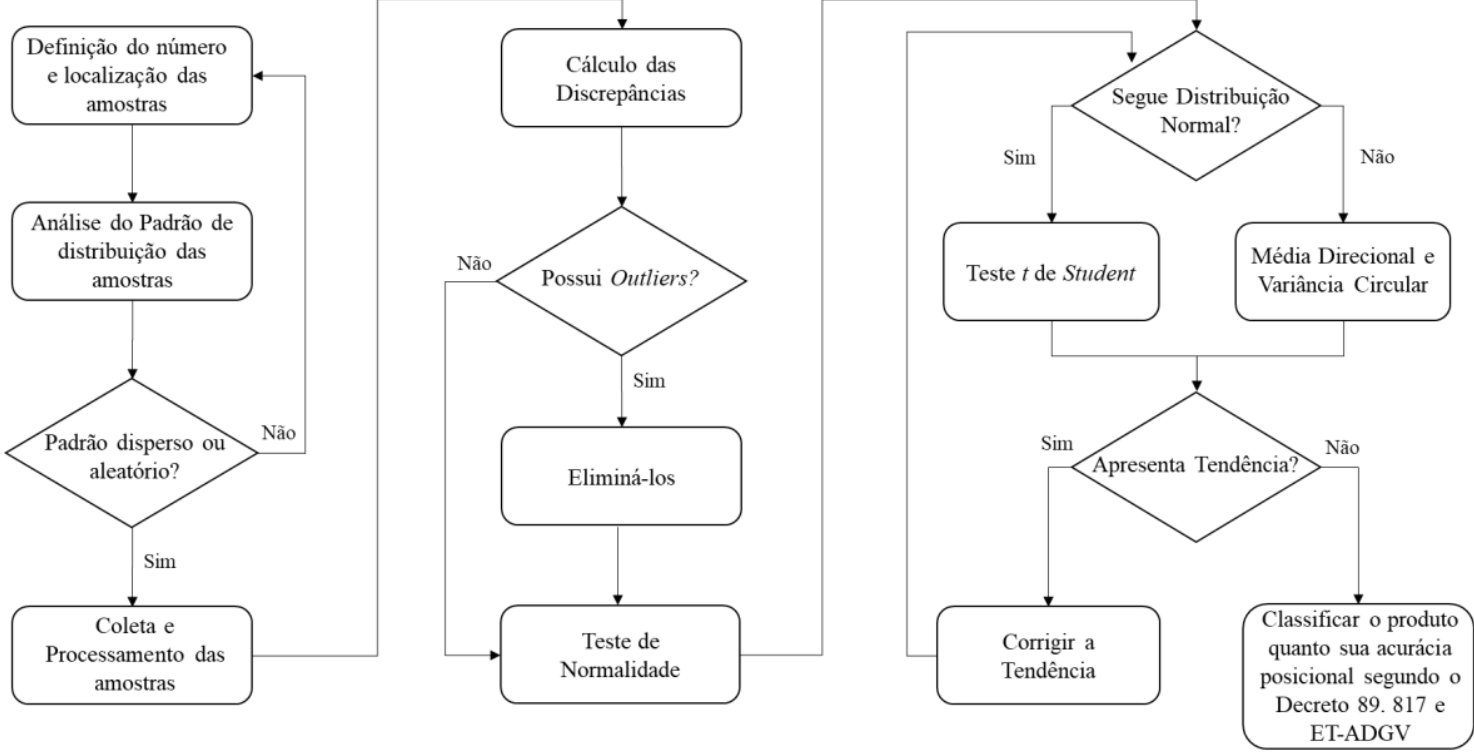

Fonte: Elaborado pelos autores.

De acordo com as normativas, o produto de teste deve ser dividido em quadrículas de $4 \mathrm{~cm}$ por $4 \mathrm{~cm}$, na escala do produto (neste caso, a escala de teste, 1/25.000). Em seguida, deve-se fazer uma inspeção em cada quadrícula gerada, de forma a detectar aquelas que possuem pelo menos um ponto identificável para análise da acurácia posicional. Neste estudo, 251 quadrículas foram consideradas válidas, já que apresentaram um ponto bem identificado no produto e em campo.

Apesar da ET-CQDG (DSG,2016) sugerir o uso de um nível de inspeção médio (nível II) e LQA de 4\%, o que resultaria para a perspectiva de Lote 
Isolado um QL de 20\% e um tamanho amostral de 20 pontos. Neste trabalho, para ser mais rigoroso, utilizou-se um $\mathrm{QL}=5 \%$ obtendo um número amostral de 42 pontos. Com o objetivo de se ter uma maior confiabilidade no processo de avaliação da acurácia posicional, arredondou-se este valor para cima definindo o tamanho amostral igual a 50 pontos de checagem.

Definido o número de amostras, avalia-se o padrão de distribuição espacial no produto de teste. Segundo Santos et al. (2016), distribuições espaciais agrupadas comprometem a avaliação da acurácia posicional do produto, desta forma, deve-se buscar atingir um padrão de distribuição disperso ou aleatório. Os autores sugerem a utilização da estatística espacial para a avalição da distribuição espacial, tal como a Função K de Ripley, que avalia se a distribuição espacial se mantém constante em diferentes escalas, sob o mesmo intervalo de distâncias (SANTOS et al, 2016).

Em seguida, realizou-se a coleta das amostras in loco. Merchant (1982) afirma que a acurácia dos pontos de referência deva ser de um terço do erro padrão (EP) da classe escolhida, que neste estudo foi a de 1/25.000. Algumas normas, como a STANAG 2215 (Standard from North Atlantic Treatment Organization), são mais rigorosas e definem que os pontos de referência devam ser de um quinto do EP (NSA, 2010). Para ambos os casos, esta precisão foi alcançada, visto que, a precisão planimétrica dos pontos GNSS variou de 1 a 33 centímetros.

As coordenadas dos pontos de teste e suas homólogas de referência (ambas no mesmo sistema de coordenadas) foram tabuladas e importadas para o GeoPEC v. 3.4, para a avalição da acurácia posicional.

O software GeoPEC foi desenvolvido por Santos (2010) e visa auxiliar os profissionais na realização da avaliação da acurácia posicional de produtos cartográficos de forma automática. Em sua versão 3.4, segue a metodologia descrita pela ET-CQDG (DSG, 2016) e por Santos et al. (2016), utilizando a estatística espacial para auxiliar na avaliação do produto cartográfico. $\mathrm{O}$ software também calcula e analisa as discrepâncias posicionais, usando como 
entrada de dados um arquivo de texto (.txt), contendo as coordenadas de teste e suas homólogas de referência.

A partir da comparação do posicionamento de pontos no produto de teste e seus homólogos coletados no levantamento de campo, foram calculadas as discrepâncias posicionais nas componentes ( $\Delta$ Este e $\Delta$ Norte), como mostram as Equações 1 e 2, e a discrepância posicional planimétrica $\left(D_{P}\right)$, conforme a Equação 3:

$$
\begin{gathered}
\Delta E s t e=E_{\text {ref }}-E_{\text {test }} \\
\Delta \text { Norte }=N_{\text {ref }}-N_{\text {test }} \\
D p=\sqrt{\Delta \text { Este }^{2}+\Delta \text { Norte }^{2}}
\end{gathered}
$$

Estas discrepâncias devem passar por um processo estatístico para avaliação de outliers e efeitos sistemáticos (tendência), os quais podem comprometer a classificação do produto, segundo o Decreto $n^{\circ} 89.817$ (BRASIL, 1994) e a norma ET-CQDG (DSG,2016). Além disto, tais testes estatísticos possibilitam o analista a ter um maior conjunto de informações acerca da qualidade do produto avaliado.

Santos et al. (2016) definem uma metodologia a ser aplicada antes da classificação final do produto, cujo primeiro passo é a detecção de outliers, considerados por alguns autores como falhas provenientes de erros grosseiros. Para isso, o GeoPEC aplica o teste 30, o qual utiliza um limiar de aceitação que é o triplo do Erro Padrão $\left(3^{*} \mathrm{EP}\right)$ da escala de teste, em relação à classe $\mathrm{A}$ (NERO, 2005; SANTOS, 2010).

Em sequência, é feita a análise da normalidade dos dados. Muitos testes estatísticos paramétricos, como o teste $\mathrm{t}$ de Student, dependem da normal distribuição dos dados. Para verificar a distribuição da amostra, há uma série de testes disponíveis: Cramer-von Mises, Kolmogorov-Smirnov, Lilliefors, D’Agostino, Shapiro-Wilk, Jarque-Bera (Bowman- Shelton) e Filliben. Nesta pesquisa foi utilizado o teste de normalidade Jarque-Bera (Bowman-Shelton), a um nível de significância de 5\%, implementado no GeoPEC. Esse teste de normalidade compara as diferenças entre os 
coeficientes de assimetria e curtose da amostra, em relação aos valores de uma distribuição normal. Mais detalhes do cálculo podem ser obtidos nos trabalhos: Jarque e Bera (1980) e Bowman e Shelton (1975).

Para a identificação de tendência, utiliza-se o teste t de Student quando as amostras seguem uma distribuição normal, porém, em casos de não normalidade, Santos (2010) indica a utilização da Média Direcional aliada à Variância Circular. Vale ressaltar que, caso o produto apresente tendência, pode-se aplicar nele uma translação, para correção ou minimização desses efeitos. Salienta-se que após esse processo é necessário realizar a avaliação da acurácia posicional novamente.

O teste t de Student é aplicado, tradicionalmente, observando um nível de confiança $(1-\alpha)$ igual a $90 \%(\alpha=0,10)$. Assim, a partir do número de pontos coletados, chega-se a um valor limite $\mathrm{t}(\mathrm{n}-1, \mathrm{a} / 2)$, que pode ser obtido por meio de Tabela bilateral. Se $\left|t_{\text {calculado }}\right| \leq t_{\text {tabelado }}$ para cada variável a ser analisada, pode-se inferir que o produto avaliado apresenta o valor da média das discrepâncias posicionais estatisticamente igual a zero. Desse resultado, pode-se inferir que o produto não apresenta tendências em suas coordenadas, estando livre de erros sistemáticos (MERCHANT, 1982).

Nos casos em que não ocorre normalidade na distribuição das amostras, a Média Direcional tem o objetivo de conseguir uma tendência central da direção de um conjunto de vetores de discrepâncias posicionais. Já a Variância Circular tem o propósito de verificar se a Média Direcional é significativa ou não (WONG e LEE, 2005, segundo SANTOS, 2010).

Sabe-se que a Variância Circular varia de 0 a 1 (Figura 3); valores próximos de zero indicam que os vetores analisados estão na mesma direção (presença de tendência), já os valores próximos a 1 indicam direções opostas (não há tendência) (ZANETTI et al., 2016). 
Figura 3 - Análise de tendências utilizando a média direcional e a variância circular

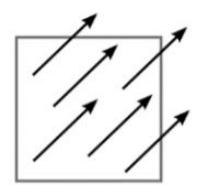

$\mathrm{Sc}=0$

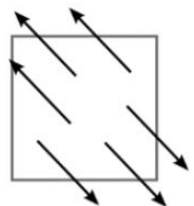

$\mathrm{Sc}=1$

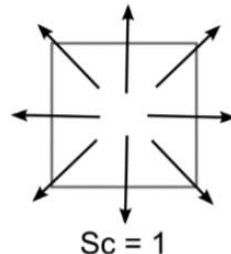

$\mathrm{Sc}=1$

Fonte: SANTOS et al. (2016).

Segundo a metodologia de Santos et al. (2016), um produto cartográfico é considerado acurado posicionalmente quando obtém uma classificação de acordo com o padrão do Decreto n 89.817 (BRASIL, 1994) e da ET-CQDG (DSG, 2016) e não apresenta tendência. Conforme metodologia descrita por esta norma, as discrepâncias devem atender aos requisitos a e b, descritos no item 2 deste trabalho. Estas duas exigências devem ser simultaneamente atingidas e, caso não sejam, deve-se alterar a escala de avaliação do produto teste ou o produto não poderá ser classificado.

\section{Resultados e discussões}

Para definir a distribuição das amostras, a área de estudo foi dividida em 50 quadrantes e uma amostra foi colocada em cada um deles. As localizações das amostras foram escolhidas conforme a metodologia do IBGE (2009), optando-se pela escolha de interseções de estradas rurais e evitando locais com obstruções dos sinais dos satélites GNSS pela vegetação e/ou edificações. Desta forma, as amostras dos cinquenta pontos de checagem foram espacializadas conforme apresentado na Figura 4. 
Figura 4 - Espacialização das amostras na região de estudo

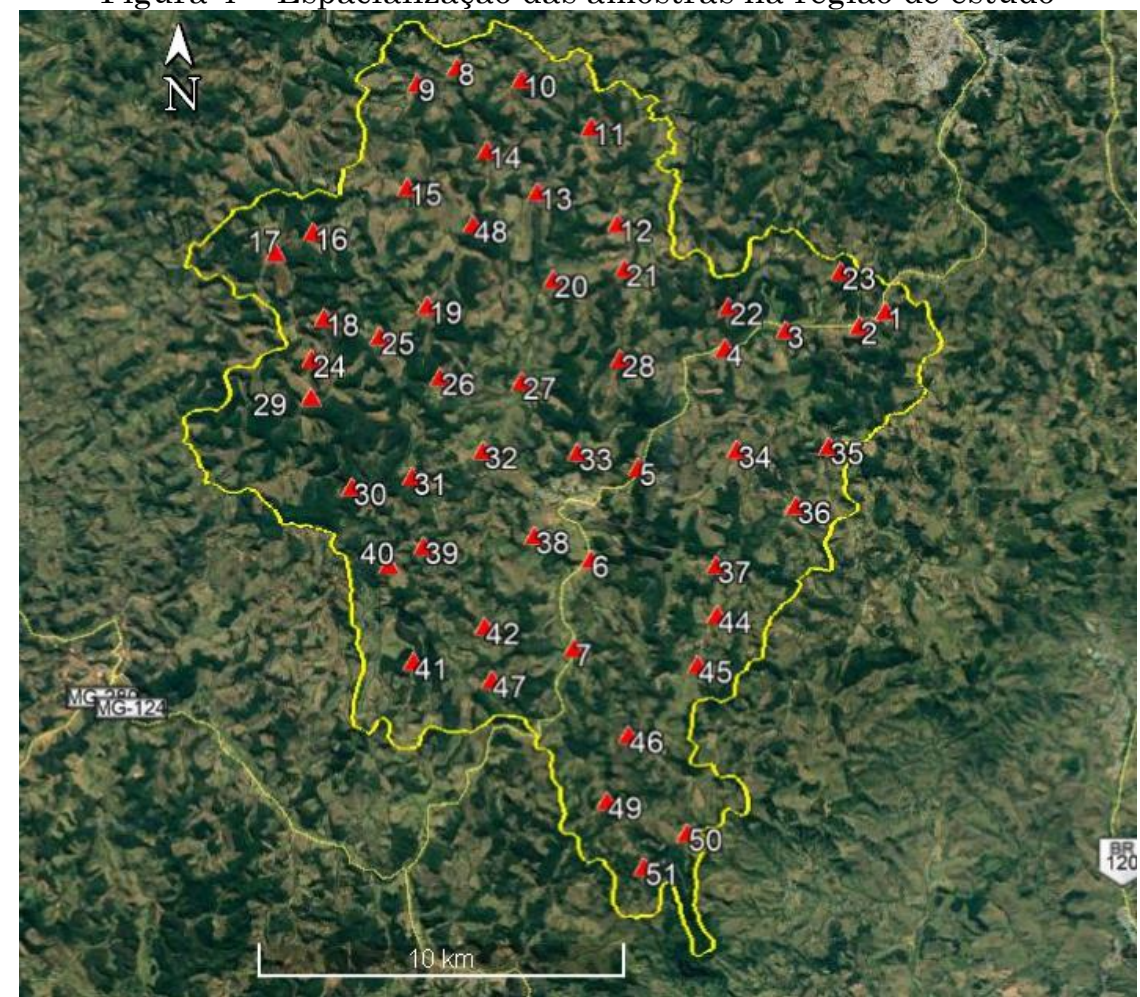

Fonte: Adaptado do Google Earth.

De posse das coordenadas da amostra de checagem, avaliou-se o padrão de distribuição das amostras através da Função K de Ripley. A Figura 5 mostra o resultado da aplicação da Função K, obtida no software ArcGIS, utilizando os cinquenta pontos de checagem. Observa-se que, até a uma distância de $2.300 \mathrm{~m}$ aproximadamente, o padrão das amostras é disperso; a partir dessa distância, a amostra apresenta um padrão de distribuição espacial aleatório. Assim, conforme recomendações de Santos et al. (2016), a amostra encontra-se bem distribuída na região de teste e pode ser utilizada para o controle de qualidade posicional. 
Figura 5 - Gráfico do padrão de distribuição espacial dos dados segundo a Função K

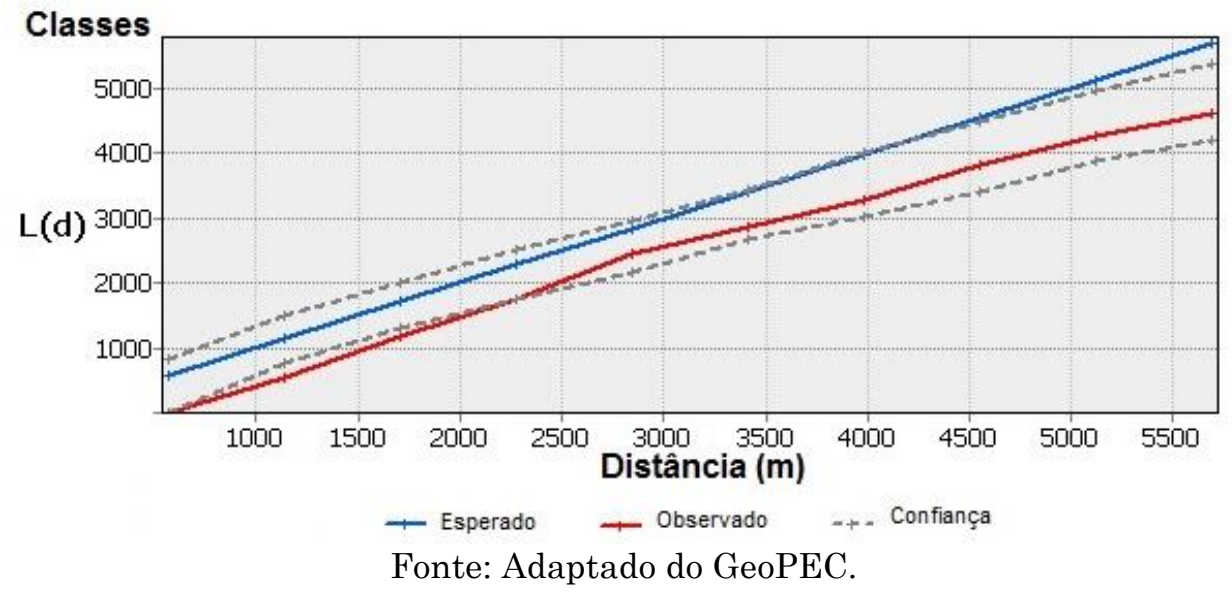

Após os cálculos das discrepâncias posicionais planimétricas de cada ponto de checagem, conforme Equações 01 a 03 , foram calculadas as estatísticas descritivas da amostra, bem como realizados os testes estatísticos de detecção de outliers, normalidade e tendência. Inicialmente, obteve-se valores de RMS igual a 3,41m, média de 3,1m e desvio padrão de $1,43 \mathrm{~m}$.

Para a detecção de outliers, utilizou-se o teste $3 \sigma$ com um limiar de 22,5 $m$ e não foi encontrado nenhum outlier na amostra de discrepâncias posicionais. Pelo método de Jarque-Bera, ao nível de significância de $5 \%$, foi constatada a normalidade dos dados, conforme apresentado na Tabela 3.

Tabela 3- Resultado do teste de normalidade Jarque-Bera

\begin{tabular}{c|c|c|c}
\hline $\mathbf{B}_{\text {cal }} \mathbf{E}$ & Bcal N & Btab & Resultado \\
\hline 0,2075 & 0,4799 & 2,86 & Amostra normal \\
\hline \multicolumn{3}{r}{ Fonte: Elaborado pelos autores. }
\end{tabular}

Para análise de tendência nas discrepâncias, realizou-se o teste $\mathrm{t}$ de Student, ao nível de significância de 10\%. Infere-se a presença de tendências tanto na componente Este quanto na componente Norte (Tabela 4). Diante deste resultado, mesmo o produto classificando como classe A (PEC-PCD na escala de 1/50.000, o mosaico do Google Earth é considerando "não acurado" posicionalmente, já que está eivado de efeitos sistemáticos.

Assim, para corrigir/minimizar esta tendência, foram realizadas duas translações no produto de teste, sendo estas translações de valores contrários 
aos das médias das discrepâncias das componentes Este e Norte, como apresentado na Tabela 7.

Tabela 4- Resultado do teste de tendência pelo teste t de Student

\begin{tabular}{c|c|c|c}
\hline Coord. & $\mathbf{t}$ tab & $\mathbf{t}$ cal & Resultado \\
\hline $\operatorname{di}(\mathrm{E})$ & 1.6794 & 2.5142 & Existe Efeito Sistemático \\
\hline $\operatorname{di}(\mathrm{N})$ & 1.6794 & -9.4856 & Existe Efeito Sistemático \\
\hline
\end{tabular}

Fonte: Elaborado pelos autores.

Após a correção da tendência, fez-se necessário realizar novamente toda a avaliação da acurácia posicional. As Tabelas 5, 6 e 7 apresentam, respectivamente, o resultado do teste de normalidade, teste de tendência e resumo das estatísticas realizadas no GeoPEC. Em sequência, a Figura 6 apresenta um gráfico das discrepâncias posicionais após a correção da tendência.

Tabela 5- Resultado do teste de normalidade Jarque-Bera após correção da tendência

\begin{tabular}{c|c|c|c}
\hline $\mathbf{B}_{\text {cal }} \mathbf{E}$ & Bcal N & Btab & Resultado \\
\hline 0,077 & 0,96 & 0,99 & Amostra normal \\
\hline \multicolumn{2}{|c}{ Fonte: Elaborado pelos autores. }
\end{tabular}

Tabela 6-Resultado do teste de tendência (teste t de Student) após correção da tendência

\begin{tabular}{c|c|c|c}
\hline Coord. & $\mathbf{t}_{\text {tab }}$ & $\mathbf{t}$ cal & Resultado \\
\hline $\operatorname{di}(\mathrm{E})$ & 1.6794 & 0,0000 & Sem Efeito Sistemático \\
\hline $\operatorname{di}(\mathrm{N})$ & 1.6794 & 0,0010 & Sem Efeito Sistemático \\
\hline \multicolumn{3}{|c}{ Fonte: Elaborado pelos autores. }
\end{tabular}

Tabela 7- Resultado das análises estatísticas

\begin{tabular}{c|c|c|c}
\multicolumn{5}{c}{ Tabela 7- Resultado das análises estatísticas } \\
\hline Estatística & Este & Norte & Posicional \\
\hline $\mathrm{n}^{\circ}$ de pontos & 50 & 50 & 50 \\
\hline média & 0,0000 & 0,0002 & 2,1722 \\
\hline desvio & 1,8474 & 1,7287 & 1,258 \\
\hline variância & 3,413 & 2,988 & 1,583 \\
\hline $\mathrm{RMS}$ & 1,8281 & 1,7106 & 2,5036 \\
\hline máximo & 3,7756 & 4,495 & 6,6881 \\
\hline mínimo & $-4,9524$ & $-3,424$ & 0,5613 \\
\hline curtose & 3,2544 & 2,5957 & 4,5254 \\
\hline assimetria & $-0,0988$ & 0,1383 & 1,1052 \\
\hline soma & 160,406 & 140,456 & 74,375 \\
\hline
\end{tabular}

Fonte: Elaborado pelos autores. 
Figura 6 - Discrepâncias posicionais observadas pelo método de feição pontual

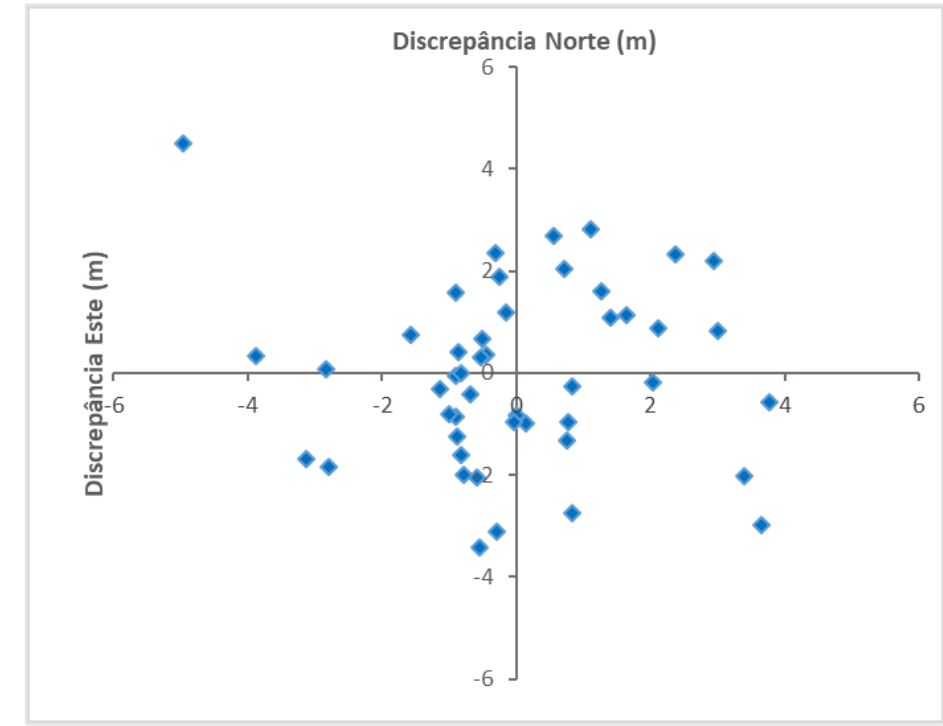

Fonte: Elaborado pelos autores.

Na Figura 7, é apresentado um mapa com a espacialização da distribuição das discrepâncias posicionais planimétricas (após correção da tendência) no município de Paula Cândido, no qual as regiões em vermelho representam os maiores valores encontrados. 
Figura 7 - Espacialização das discrepâncias posicionais no município de Paula Cândido

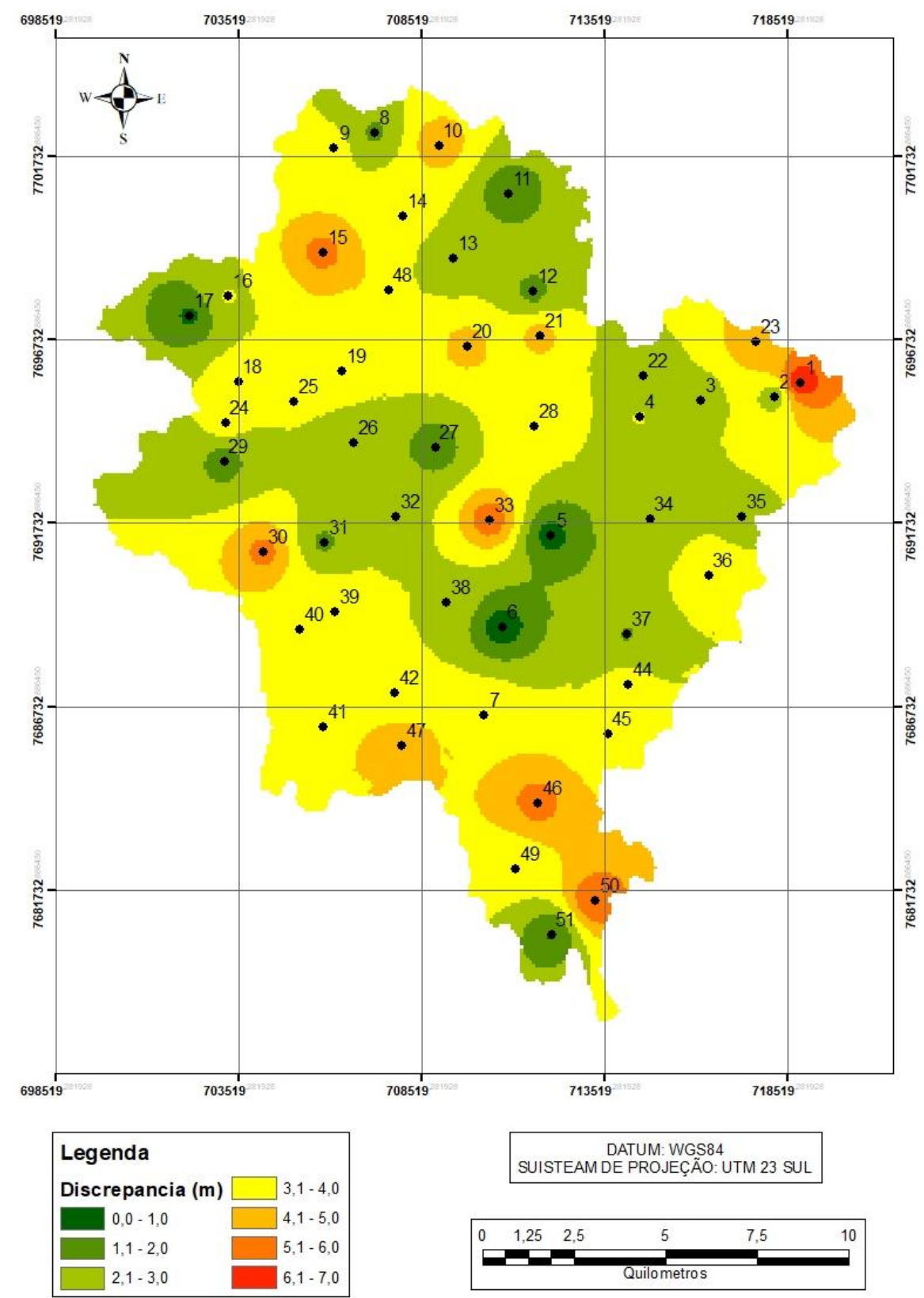

Fonte: Elaborado pelos autores.

De forma panorâmica, observa-se, por meio das Figuras 6 e 7, que a distribuição das discrepâncias foi heterogênea. Porém, ao se avaliar cada setor, constata-se que ocorreu uma concentração das menores discrepâncias no centro das imagens. Estatisticamente, 68\% das discrepâncias estão representadas no intervalo de -2 metros a 2 metros.

O produto em estudo foi considerado Classe A para a escala de 1/25.000 e Classe B para a escala de 1/10.000. Percebe-se que, mais uma vez, a 
classificação foi diferente das demais, apresentando a variabilidade da qualidade posicional do Google Earth. Apesar de as metodologias de avaliação da qualidade serem diferentes, tanto no teste aplicado quanto na quantidade de pontos de teste, os resultados obtidos se assemelham aos dados obtidos por Oliveira et al. (2009) e por Soares et al. (2010). Os resultados evidenciam que a acurácia posicional em regiões menos desenvolvidas é inferior que a das regiões mais desenvolvidas, como apontam os resultados de Silva e Nazareno (2009) para o município de Goiânia-GO. Com base nos dados da Tabela 7, o valor do RMS posicional foi superior ao EP esperado para a classe A na escala 1/10.000, e nem 70\% das discrepâncias foram inferiores ao PEC da referida escala. Destaca-se que o Decreto nº 89.817 (BRASIL, 1994) e a ET-ADGV (DSG, 2011) exigem que pelo menos 90\% das discrepâncias sejam inferiores ao PEC.

\section{Conclusões}

O mosaico das imagens disponibilizadas pelo Google Earth, compreendendo uma área de 268,39 km² da região de Paula Cândido/MG e após correção dos efeitos sistemáticos, apresentou uma classificação compatível com a classe A (PEC-PCD), para a escala 1/25.000, e com a classe B (PEC-PCD), para a escala 1/10.000, conforme o padrão brasileiro de acurácia posicional, estabelecido no Decreto nº 89.817 (BRASIL, 1994) e na ET-CQDG (DSG, 2016). Foi possível verificar que as imagens e dados advindos do Google Earth apresentaram tendências no conjunto de discrepâncias posicionais, fazendo-se necessário a correção delas, por meio da aplicação de translação, corrigindo o efeito sistemático presente.

Este trabalho buscou apresentar o potencial e a viabilidade da utilização do Google Earth para a elaboração de uma base cartográfica municipal, para municípios de pequeno porte. Considerando que a acurácia posicional da ferramenta apresentou bons resultados, compatíveis com as 
escalas do mapeamento sistemático brasileiro, conclui-se que existe a viabilidade de sua utilização.

É necessário destacar, no entanto, que esta pesquisa analisou apenas a acurácia posicional do produto da Google. Nesse sentido, ressalta-se a importância de se avaliar outros parâmetros de qualidade que influenciam na criação de uma base cartográfica.

Não existe um parâmetro que define qual deve ser a escala para uma cartografia municipal, porém as escalas encontradas neste estudo são comumente utilizadas em mapeamento de áreas de grande extensão. Dessa forma, ressalta-se que a utilização do Google Earth para a atualização do mapeamento sistemático brasileiro é uma possibilidade, embora sua utilização deva ser analisada com base nas particularidades de cada região. Essa análise é necessária porque, ainda que para este estudo o mosaico para a região tenha apresentado escala compatível ao mapeamento sistemático, após correção da tendência; diversas pesquisas considerando outras regiões mostraram resultados opostos.

Nesse contexto, para a atualização do mapeamento sistemático brasileiro, por meio de informações do Google Earth, faz-se necessária uma avalição em escala estadual, e até mesmo nacional, pois cada região apresenta um tipo de mosaico. Conclui-se, portanto, que a viabilidade de utilização do Google Earth só pode ser determinada após prévia avaliação da acurácia posicional da ferramenta para a região específica.

Recomenda-se, para trabalhos futuros, a investigação da acurácia posicional através de feições lineares extraídas no Google Earth e a avaliação do potencial para restituição, visto que seu mosaico de imagens é muito variável. 


\section{Agradecimentos}

Este estudo contou com o incentivo da Universidade Federal de Viçosa, por meio do Departamento de Engenharia Civil (DEC) e do Programa de PósGraduação em Engenharia Civil, que disponibilizaram seus equipamentos, instalações e aplicativos computacionais.

O presente trabalho foi realizado com apoio da Coordenação de Aperfeiçoamento de Pessoal de Nível Superior - Brasil (CAPES) - Código de Financiamento 001.

\section{Contribuição dos autores}

Prof. Dr. Afonso de Paula: realizou a orientação e supervisão do projeto.

Raiane Rintielle: doutoranda, uma das responsáveis pelo projeto, participou da coleta dos dados, análise, discussão e confecção do manuscrito.

Matheus Henrique: doutorando, realizou o planejamento, bem como a coleta dos dados, processamento, análise dos resultados e confecção do manuscrito.

Jairo Dias: mestrando, participou da confecção do manuscrito.

\section{Referências}

ARIZA, F. J. Calidad En La Producción Cartográfica. Ed. Ra-Ma, Madrid, Espanha, 2002. 424 p.

BOWMAN, K.; SHELTON, L. Omnibus test contours for departures from normality based on $b 1$ and b2. Biometrika. vol. 62, 1975. pp. 243-250.

BRASIL. Decreto n 89.817 de 20 de junho de 1984. Normas Técnicas da Cartografia Nacional. Diário Oficial da União, 20 de junho de 1984.

BRAVO, J. V. M.; SLUTER, C. R. O problema da qualidade de dados espaciais na era das informações geográficas voluntárias. Boletim de Ciências Geodésicas (Online), vol. 21, 2015. pp. 56 - 73. 
CINTRA, J. P.; NERO, M. A. Metodologia para controle de qualidade de mapeamentos sistemáticos em meio digital. XXII Congresso Brasileiro de Cartografia. Macaé - RJ, 2005. pp. 1 -14.

DSG. Especificação Técnica para a Aquisição de Dados Geoespaciais Vetoriais (ET-ADGV). Diretoria do Serviço Geográfico. Brasília-DF. 2011. Site <http://www.geoportal.eb.mil.br/images/PDF/ET_ADGV_Vs_2_1_3.pdf>, acessado em setembro de 2017.

DSG. Norma da Especificação Técnica Para Controle de Qualidade de Dados Geoespaciais (ET-CQDG). Diretoria do Serviço Geográfico. BrasíliaDF. 2016. Site <http://www.geoportal.eb.mil.br/images/PDF/ET_CQDG _1a_edicao_2016.pdf>, acessado em setembro de 2017.

GOODCHILD, M. F. Foreword. In: SHI, W. (ed.) Principles of Modeling Uncertainties in Spatial Data and Spatial Analyses. CRC Press, Boca Raton, Flórida, EUA, 2010. 432 p.

GALO, M.; CAMARGO, P. de O. O uso do GPS no controle de qualidade de cartas. $\mathbf{1}^{\mathbf{0}}$ Congresso Brasileiro De Cadastro Técnico Multifinalitário-COBRAC, Florianópolis, 1994, pp. 41-48.

IBGE. Avaliação Planialtimétrica de Dados ALOS/PRISM. Estudo de Caso: Itaguaí (RJ). Relatório 2009. Instituto Brasileiro de Geografia e Estatística. Rio de Janeiro, 2009. 32p.

IBGE. Manuais Técnicos em Geociências, número 13, Avaliação da Qualidade de Dados Geoespaciais. Coordenação de Cartografia. Instituto Brasileiro de Geografia e Estatística. Rio de Janeiro, 2017. 93p.

JARQUE, C. M.; BERA, A. K. Efficient tests for normality, homoscedasticity and serial independence of regression residuals. Economics Letters, Elsevier, vol. 6, 1980. pp. 255-259.

LOPES, E. E. Proposta Metodológica Para Validação De Imagens De Alta Resolução Do Google Earth Para A Produção De Mapas. Dissertação de mestrado. Universidade Federal de Santa Catarina, Programa de PósGraduação em Engenharia Civil, Florianópolis - SC, 2009. 112p.

MERCHANT, D. C. Spatial Accuracy Standards for Large Scale of Maps. Proceedings of the technical congress on surveying and mapping, Stanford University, Stanford, California, 1982. pp. 222-231. 
NERO, M. A. Proposta para o controle de qualidade de bases cartográficas com ênfase na componente posicional. Tese de doutoramento. Escola Politécnica da Universidade de São Paulo, Programa de Pós-Graduação em Engenharia de Transportes, São Paulo, 2005. 186 p.

NOGUEIRA Jr, J. B. Controle de Qualidade de Produtos Cartográficos: Uma Proposta Metodológica. Dissertação de mestrado. Universidade Estadual Paulista. Programa de Pós-Graduação em Ciências Cartográficas, Presidente Prudente, 2003. 143p.

NSA. Evaluation of land maps, aeronautical charts and digital topographic data. STANAG (Standardization Agreement) $\mathrm{n}^{\circ} 2215$ (Edition 7). Tex not published, NATO Standardization Agency,Bruxelas, 2010. 29p.

OLIVEIRA, M. Z.; VERONEZ, M. R.; TURANI, M.; REINHARDT, A. O. Imagens do Google Earth para fins de planejamento ambiental: uma análise de exatidão para o município de São Leopoldo/RS. Anais do XIV Simpósio Brasileiro de Sensoriamento Remoto, Natal, 2009. pp. 1835-1842.

PAIVA, C. C. A contemplação do mundo. O Google Earth, a Comunicação e a Terra digitalizada. Anais do XXXII Congresso Brasileiro de Ciências da Comunicação. Rio de Janeiro, 2010. Pp.1 -15. Site: $<$ http://www.bocc.uff.br/pag/bocc-contempl-paiva.pdf $>$, acessado em outubro de 2017.

SANTOS, A. P. Avaliação da Acurácia Posicional em Dados Espaciais com o uso da Estática Espacial. Dissertação de mestrado. Universidade Federal de Viçosa, Programa Pós-Graduação em Engenharia Civil, Viçosa, 2010. 110p.

SANTOS, A. P.; RODRIGUES, D. D.; SANTOS, N. T.; GRIPP JR. J. Avaliação da acurácia posicional em dados espaciais utilizando técnicas de estatística espacial: proposta de método e exemplo utilizando a norma brasileira. Boletim de Ciências Geodésicas. Curitiba, vol. 22, nº 4, 2016. pp. 630 - 650 .

SANTOS, A. P. Software GeoPEC versão 3.4. Viçosa - MG, 2017.

SANTOS, C. Leituras Geográficas e Cartográficas nos Contextos

Educacionais. Editora Clube de Autores, Nova Iguaçu - RJ. 2013. 124 p.

SCHMITT, A.; ROSENFELDT, Y. A. Z.; OLIVEIRA, M. O.; ROSOLEM, G. P. N.; LOCH, C. Proposta de mobilidade coletiva para a Região Metropolitana de Florianópolis pensada a partir de imagens do Satélite GeoEye e banco de dados 
de software SIG. Anais do XVI Simpósio Brasileiro de Sensoriamento Remoto, Foz do Iguaçu - PR, 2013. pp. 0810 - 0817.

SILVA, L. A.; NAZARENO, N, R, X. Análise do padrão de exatidão cartográfica da imagem do Google Earth tendo como área de estudo a imagem da cidade de Goiânia. Anais do XIV Simpósio Brasileiro de Sensoriamento Remoto, Natal-CE., 2009. pp. 1723-1730.

SOARES, M. C; RUARO, T. A; AGUIAR, C. R. Controle De Qualidade Da Base Cartográfica Da Cidade De Pato Branco No Software Google Earth. Synergismus scyentifica UTFPR, I Semana Acadêmica de Agrimensura, Pato Branco-PR, 2010. pp. 28 - 30.

TOMMASELLI, A. M. G.; MONICO, J. F. G.; CAMARGO, P. O. Análise da exatidão cartográfica da carta imagem. Anais do V Simpósio Brasileiro De Sensoriamento Remoto, Natal, 1988, pp. 253-257.

VERGARA, O. R.; CINTRA, J. P.; D'ALGE, J. C. L. Avaliação da exatidão cartográfica de documentos atualizados com imagens orbitais e sistemas de informação geográfica. Anais do XX Congresso Brasileiro de Cartografia. Porto Alegre - RS, 2001. Pp. 1 - 7.

WONG, D.W.S; LEE, J. Statistical Analysis of Geographic Information with ArcView GIS and ArcGIS. John Wiley \& Sons. 2005. 464 p.

ZANETTI, J., PAUlA, R. M., SANTOS. A. P., MEDEIROS, N.G. Avaliação da acurácia posicional planimétrica de ortoimagens disponibilizadas nos sistemas de informações geográficas. Revista Brasileira de Cartografia, Rio de Janeiro, N 68/7, 2016. pp. 1341-1352. 